\title{
A Rapid Histological Score for the Semiquantitative Assessment of Bone Metastases in Experimental Models of Breast Cancer
}

\author{
Marcus Neudert ${ }^{a}, b \quad$ Christian Fischer $^{a}$ Burkhard Krempien ${ }^{c} \quad$ Markus J. Seibel $^{\mathrm{a}, \mathrm{d}}$ Frieder Bauss $^{\mathrm{e}}$ \\ a Department of Medicine, University of Heidelberg, \\ ${ }^{b}$ Department of Otorhinolaryngology, University of Dresden, \\ c Department of Pathology, University of Heidelberg, Germany \\ ${ }^{d}$ Bone Research Program, ANZAC Research Institute, The University of Sydney, Australia \\ e Roche Diagnostics GmbH, Pharma Research, Penzberg, Germany
}

Key Words

Bone metastases - Bisphosphonates - Tumor osteolysis . Histology · Score

\section{Summary}

Background: Using a nude rat model of site-specific metastatic bone disease (MBD), we developed a semiquantitative histological score for rapid assessment of lytic lesions in bone. This provides additional information to conventional histological measurement by clarifying the extent and location of metastatic infiltration and the tumor growth pattern. The score can also be used to assess the action of bisphosphonates on bone metastases. Materials and Methods: Male nude rats ( $n=12$ per group) were inoculated with the human breast cancer cell line MDA-MB-231 via the femoral artery. Following appearance of radiographically visible osteolytic lesions on day 18 , the animals received phosphate-buffered saline (PBS; controls) or ibandronate (IBN, $10 \mu \mathrm{g} \mathrm{P} / \mathrm{kg}$ ) daily until day 30 . Whole body radiographs were obtained on days 18 and 30, and osteolytic areas (OA) were determined by radiographic computer-based analysis (CBA). On day 30, MBD was assessed in both tibias using conventional histological CBA and the new scoring system. Results: Metastatic tumor area correlated with the total sum of the new score in both PBS- $(r=0.762)$ and IBN-treated animals $(r=0.951 ; p<0.001)$. OA correlated well with the total sum in both groups $(r=0.845$ and 0.854 , respectively; $p<$ 0.001). Conclusion: Significant reduction of bone marrow and cortical infiltration of tumor cells with IBN suggested local control of metastases.
Schlüsselwörter

Knochenmetastasen - Bisphosphonate - Tumorosteolyse . Histologie · Graduierungssystem

\section{Zusammenfassung}

Hintergrund: Mit Hilfe eines etablierten Tiermodells zur Erzeugung lokalisationsspezifischer Knochenmetastasen in der Nacktratte wurde ein semiquantitatives histologisches Graduierungssystem zur schnellen Bewertung osteolytischer Knochenmetastasen entwickelt. Das Graduierungssystem liefert hinsichtlich der Metastasenlokalisation, deren Ausmaß und Infiltrationsmuster wertvolle Zusatzinformationen zu den konventionellen histologischen Untersuchungsmethoden. Damit kann beispielsweise auch die pharmakologische Wirkung von Bisphosphonaten auf die Knochenmetastasierung beurteilt werden. Material und Methoden: Männlichen Nacktratten ( $\mathrm{n}=12$ pro Gruppe) wurden Zellen der humanen Brustkrebszellinie MDA-MB-231 in die Oberschenkelarterie inokuliert. Ab dem Auftreten radiologisch erkennbarer Osteolysen 18 Tage nach Inokulation erhielten die Tiere bis zum Studienende (Tag 30) täglich entweder eine subkutane Applikation einer Phosphat-Puffer-Lösung (Kontrollgruppe) oder lbandronat (IBN, $10 \mu \mathrm{g} \mathrm{P} / \mathrm{kg}$; Behandlungsgruppe). Konventionelle Röntgenaufnahmen wurden an den Tagen 18 und 30 nach Tumorinokulation angefertigt und die Osteolysenflächen mittels Computerauswertung bestimmt. Nach Studienende wurde der Metastasenbefall in beiden Tibiae sowohl konventionell histologisch als auch mittels des neuen Graduierungssystems ausgewertet. Ergebnisse: Die Metastasenfläche korrelierte mit der kummulativen Punktsumme des Graduierungssystems sowohl in der Kontrollgruppe $(r=0,762 ; p<0,001)$ als auch in der IbandronatGruppe $(r=0,951 ; p<0,001)$. Ebenso war die Osteolysenfläche eng mit der Punktesumme in beiden Gruppen korreliert ( $r=0,845$ und 0,$854 ; p<0,001)$. Schlussfolgerung: Die signifikante Reduktion von Knochenmark- und Kortikalisbefall durch IBN deuten auf eine gute lokale Kontrolle des Metastasenwachstums hin.

\begin{tabular}{|c|c|}
\hline KARGER & (C) 2008 S. Karger GmbH, Freiburg \\
\hline $\begin{array}{l}\text { Fax +49 } 7614520714 \\
\text { Information@Karger.de } \\
\text { www.karger.com }\end{array}$ & $\begin{array}{l}\text { Accessible online at: } \\
\text { www.karger.com/onk }\end{array}$ \\
\hline
\end{tabular}




\section{Introduction}

Bone metastases have significant impact on the prognosis and quality of life of cancer patients, and their treatment is an important area of cancer care [1-3]. Preclinical evaluation of drugs is a crucial step in the development of cancer treatments, and has supported the widespread use of bisphosphonates in managing the skeletal complications of metastatic bone disease [4]. The assessment of metastatic tumor burden is a prerequisite for such analyses. Experimental models for metastatic bone disease (MBD) and their outcomes are currently assessed by radiographic and histological quantification of bone destruction. Conventional histomorphometry remains the current gold standard for assessing bone lesion status. However, this approach can be time-consuming, requires considerable technical resources, and is not possible for advanced tumors.

Krempien et al. [5] have described a basic, 4-stage scoring system for the histological evaluation of bone lesions in an animal model [5]. This score, ranging from normal bone histology (0) to severe $(+++)$ bone lesions, is a concise assessment tool but is unable to provide information on metastatic growth patterns, which is necessary for a detailed assessment of skeletal destruction. As for conventional histomorphometry, this system is also less useful once the tumor has destroyed a significant proportion of bone. For advanced bone lesions, alternative criteria to evaluate metastatic burden have been developed, including complex 3-dimensional microtomographic analyses [5-7]. However, while these techniques yield a wealth of detail, they are not widely available and do not promote high-throughput screening of potentially useful drugs.

We have developed a rapid, semiquantitative histological score that could be used in addition to conventional histological methods. Our aim was to identify succinct parameters for the comprehensive analysis of patterns of osteolytic destruction and tumor cell infiltration, and to delineate the action of bisphosphonate treatment on bone metastases. The present study describes the proposed scoring system and its validation against standard techniques in an animal model of site-specific metastatic osteopathy [8]. A key goal was to ensure that the new score should directly reflect quantitative evaluation of osteolyses, and still be informative when standard histomorphometric techniques are not applicable (e.g. when bone destruction is far advanced).

\section{Material and Methods}

\section{Animals}

A total of 24 athymic male nude rats (7-8 week old rnu/rnu Rowett nudes; Harlan-Winkelmann, Borchen, Germany) were housed under specific pathogen-free conditions. All animals had free access to tap water and were fed ad libitum with a commercial standard feed (Sniff Spezialdiäten, Soest, Germany). Experiments were approved by the governmental Animals Ethic Committee (Regierungspräsidium Karlsruhe, Germany).
Inoculation of Tumor Cells

Inoculation was achieved as described previously by Neudert et al. [8] and Cailleau et al. [9]. In brief, $10^{5}$ cells of the human breast cancer cell line MDA-MB-231 (kindly provided by Dr. Theresa Guise, University of Texas, San Antonio, USA) were suspended in $0.5 \mathrm{ml}$ of phosphatebuffered saline (PBS) and injected into the femoral arteries of both legs via a polyethylene catheter (approximately $0.1 \mathrm{~mm}$ external diameter). On average, osteolytic bone metastases became visible on hind limb radiographs by day 18 post-inoculation (p.i.). All procedures were performed under general anesthesia.

\section{Experimental Protocol}

Inoculated rats were divided into 2 groups of 12 animals each. Following the appearance of radiographically visible osteolytic lesions on day 18 , each group was injected subcutaneously with PBS (control) or ibandronate (IBN; Roche Diagnostics GmbH, Mannheim, Germany) daily for 12 days (to day 30 p.i.). IBN was dissolved in isotonic saline (adjusted to $\mathrm{pH} 7.4$ ) to give $10 \mu \mathrm{g} \mathrm{P} / \mathrm{kg} /$ day, which corresponds to $51.4 \mu \mathrm{g}$ of free acid equivalents. This dose prevents tumor-related bone destruction in rats [10]. Whole body radiographs were taken on days 18 and 30. On day 30, the animals were sacrificed, and both tibias were removed and processed for histological analyses, specifically to measure the metastatic area (MA). Osteolytic areas (OA) were quantified using radiographic computerbased analysis (X-CBA), as described below. Tumor extent at study termination was determined by histological computer-based analysis (H-CBA). Metastatic deposits were evaluated using the new scoring system.

\section{Radiographic Computer-Based Analysis}

Whole body radiographs were taken during general anesthesia of the animals who were placed in an anterior-posterior orientation on a high-resolution mammography film (Strukturix D4 DW ETE $18 \times 24 \mathrm{~cm}$, AGFA, Köln, Germany). X-ray exposure ( $55 \mathrm{kV}, 5 \mathrm{~mA}$ for $90 \mathrm{~s}$ ) occurred in a radiographic animal inspection unit (Torrex 120D, EG and G Astrophysics Research, Long Beach CA, USA). Radiographs were scanned (resolution of $480 \mathrm{dpi}$ ) and analyzed by 2 independent investigators blinded to the protocol. Analyses were performed using specialist software at a magnification of $\times 300$ (CBA program, CBA 8000, Leica QWin Pro V2.0, Leica, Bensheim, Germany). Osteolytic foci $>0.5 \mathrm{~mm}$ in diameter (detection limit) were recognized as radiolucent lesions and manually delineated to determine the number $(\mathrm{n})$, perimeter $(\mathrm{mm})$, and OA $\left(\mathrm{mm}^{2}\right)$. Since initially separate lesions showed a strong tendency to merge during tumor growth, only the total OA per bone and animal was used for further analyses. Animals were then stratified into 2 groups for further analysis on day 30 p.i. using OA measured on day 18 p.i. as the critical parameter. Since smaller lesions were more likely to respond to IBN treatment than advanced ones, an empirical cut-off of $6 \mathrm{~mm}^{2}$ was established based on OAs measured on day 18.

\section{Histological Computer-Based Analysis}

On day 30 p.i., local metastatic bone destruction was measured by histology in both tibias. Bones were separated from surrounding tissue and fixed in $10 \%$ neutral buffered formalin, and subsequently decalcified in a $14 \%$ EDTA solution. Following conventional processing and paraffin embedding, sections $(200 \mu \mathrm{M})$ were cut in the anterior, median, and posterior third of each bone. Following staining with Masson-Goldner, sections showing maximum tumor extent were selected and used to determine MA. Metastatic foci were counted and their respective areas measured by manual delineation (final magnification $\times 400$ ). Results (number and area) were processed using specialized software (CBA 8000, Leica QWin Pro V2.0). All histological examinations were performed by 2 independent investigators blinded to the protocol.

\section{Semiquantitative Histological Analysis}

Bone sections used for the quantification of absolute MA were also employed for the semiquantitative evaluation using the new scoring system. 


\section{Area and topography of metastatic infiltrated bone}


Fig. 1. Evaluation of metastatic bone involvement using the new score system. To estimate tumor area (A), proximal tibias were virtually divided into 4 equal quarters and the metastatic area assessed for each (see diagram top right). Each quarter with lesions scored 1 point (e.g., 2 a). In comparison 2, 3, and 4 points were accrued by $2 \mathrm{~b}, 2 \mathrm{c}$, and $2 \mathrm{~d}$, respectively. For topographical criteria of infiltration (TC, TM, TE), complete destruction of cortical bone (TC) on one side is shown in 2 a ( 2 points), completely destroyed cortical structure on both sides is shown in $2 \mathrm{c}$ and $2 \mathrm{~d}$ ( 3 points). The medullary canal is infiltrated in all sections in this figure; TM therefore scored 1 in each case. Examples of the extent of epiphyseal involvement (TE) are shown in $2 \mathrm{e}, 2 \mathrm{f}$, and $2 \mathrm{~g}$. A metastatic lesion growing directly distal of the epiphysis causing part destruction of the growth plate is show in $2 \mathrm{e}$ (scoring 1 point). The same section is presented at a higher magnification $(\times 40)$ in $2 \mathrm{f}$. Complete destruction of the growth plate and consecutive infiltration of the epiphysis (scoring 2 points) is shown in $2 \mathrm{~g}$ (magnification $\times 25$ ).

The key output of this scoring system is the sum $(\Sigma)$ of a number of distinct variables: tumor area (A), certain topographic indices (T), and the mode of infiltrative tumor behavior (Q). This system attempts to reflect the natural heterogeneity of bone metastases and include a full evaluation of lesion status. This requires measurement of the extent and the specific localization of metastatic infiltration. Osteolytic lesions typically first occur in the upper half of the tibia. Therefore, histological sections were bisected longitudinally with the proximal half used for analyses.

\section{Defining the Score Components}

Three main components were included in this analysis:

i) Infiltrated area (A): In order to estimate the relative proportion of metastatic invasion in each section and to allow comparisons between samples of different total bone area, each section was divided into 4 virtu-
Table 1. Overview of the scoring system

\begin{tabular}{lll}
\hline Parameter & Score & Interpretation \\
\hline $\begin{array}{l}\text { A: tumor area (invaded quarters } \\
\text { of bone) }\end{array}$ & $1-4$ & \\
$\begin{array}{l}\text { T: topography of invaded bone } \\
\text { TC (Cortex) }\end{array}$ & & \\
& 1 & not affected \\
& 2 & invaded \\
TM (Bone marrow) & 3 & destructed on one side \\
& 0 & not affected sides \\
TE (Epiphysis) & 1 & invaded \\
& 0 & not affected \\
Q: Qualitative tumor growth & 1 & invasion of growth plate \\
& 2 & invasion of epiphysis \\
& 2 & well delineated/encapsulated \\
\end{tabular}

al quadrants. Regardless of their number and position within the section, all metastatic foci were assessed as though they comprised 1 cumulative lesion. The area of this cumulative lesion was then estimated with reference to the size of 1 quadrant for that section. Scale bars and quantitative estimates of lesion areas are therefore redundant for this analysis. For example, if the total area of the combined foci was estimated to cover 1 quadrant (i.e., $25 \%$ of the total area) then this scored 1 ; if the total area of combined lesions was $50 \%$ of the section (i.e., 2 quadrants) then this scored 2. Total destruction of bone would therefore score a maximum of 4. Examples of how the scoring was performed are provided in figure 1.

ii) Topographical criteria of infiltration (T-indices): In order to estimate the infiltration pattern of metastases, topographic localization to cortical bone (TC), bone marrow (TM), and the invasion and/or infiltration of the epiphyseal growth plate (TE) were evaluated separately. Scoring criteria are summarized in table 1. Cortical bone (TC): In long bones, tumor-derived osteolytic lesions typically begin in the medullary canal and eventually extend to the endosteal surface of the cortex. This results in a reduction in structural integrity and increased radiolucency [11]. Intact bones, with no histological signs of cortical metastatic infiltration were scored 0 . In contrast, regions of cortical bone already destroyed by perforating lesions exhibit higher radiolucency. These regions were scored 1-3: $1=$ slightly infiltrated cortex; 2 = completely destroyed cortical structure on one side; and 3 = destruction of both cortices of the section. Bone marrow (TM): In contrast to cortical sites, widespread infiltration of bone marrow does not induce equivalent lesions on radiographs. Consequently, when bone marrow was affected (alone or in combination with other sites of lesion), 1 scoring point was added. Epiphyseal growth plate (TE): Small metastatic lesions preferentially grow distal to the epiphyseal growth plate of the proximal tibia. One point was scored for epiphyseal infiltration by malignant cells. Tumors not only affecting the growth plate but also the epiphysis scored an additional 2 points.

iii) Quality of infiltration pattern $(\mathrm{Q})$ : The general appearance of tumor growth patterns is heterogeneous. It is important to differentiate between well-delineated and localized metastatic infiltration and diffuse and invasive lesions. Localized and confined lesions accumulated a lower score (1 point) than diffuse invasive metastases (2 points). Both single parameters (A, T, and Q scores) and the total cumulative score sum of all parameters were used for correlation analysis with the absolute OA and MA determined by X-CBA and H-CBA, respectively. All parameters were expressed per affected tibia per animal.

\section{Statistical Analyses}

Statistical analyses were performed using the SAS software package (SAS Institute, Cary, NC, USA). Descriptive data are expressed as mean \pm stan- 
Table 2. Radiographic $(\mathrm{X})$ and histological $(\mathrm{H})$ parameters determined by computer-based analyses (CBA) or semiquantitative scoring obtained on day 30 p.i.; data are mean \pm standard deviation and expressed in absolute values $\left(\mathrm{mm}^{2}\right)$ or score; osteolytic $(\mathrm{OA})$ and metastatic areas (MA) are shown for the whole groups (total) and after stratification for initial OA on day 18 p.i. $\left(<6 \mathrm{~mm}^{2}\right.$ and $>6 \mathrm{~mm}^{2}$, respectively as explained in the text)

\begin{tabular}{|c|c|c|c|c|c|c|c|c|c|}
\hline & $\begin{array}{l}\text { Osteolyses } \\
(\mathrm{OA}) \\
\mathrm{X}-\mathrm{CBA}, \mathrm{mm}^{2}\end{array}$ & $\begin{array}{l}\text { Metastases } \\
\text { (MA) } \\
\text { X-CBA, } \mathrm{mm}^{2}\end{array}$ & $\begin{array}{l}\text { Score } \\
\text { A }\end{array}$ & $\mathrm{TC}$ & $\mathrm{TM}$ & $\mathrm{TE}$ & $\Sigma \mathrm{T}$ & Q & score $\Sigma$ \\
\hline \multicolumn{10}{|l|}{ Controls (PBS) } \\
\hline Total & $9.07 \pm 5.02$ & $6.5 \pm 6.3$ & $1.71 \pm 1.31$ & $1.76 \pm 1.03$ & $0.71 \pm 0.47$ & $0.47 \pm 0.80$ & $2.94 \pm 2.00$ & $1.38 \pm 0.72$ & $5.76 \pm 3.91$ \\
\hline$<6 \mathrm{~mm}^{2}(\mathrm{n}=5)$ & $7.77 \pm 5.01$ & $5.91 \pm 6.86$ & $1.50 \pm 1.43$ & $1.50 \pm 1.18$ & $0.60 \pm 0.52$ & $0.50 \pm 0.85$ & $2.60 \pm 2.22$ & $1.11 \pm 0.78$ & $5.10 \pm 4.36$ \\
\hline$>6 \mathrm{~mm}^{2}(\mathrm{n}=6)$ & $11.34 \pm 4.44$ & $7.37 \pm 7.28$ & $2.00 \pm 1.15$ & $2.14 \pm 0.69$ & $0.71 \pm 0.49$ & $0.43 \pm 0.79$ & $3.28 \pm 1.70$ & $1.43 \pm 0.53$ & $6.71 \pm 3.25$ \\
\hline \multicolumn{10}{|l|}{ IBN } \\
\hline Total & $3.80 \pm 3.42$ & $8.3 \pm 10.6$ & $1.50 \pm 1.71$ & $1.50 \pm 1.46$ & $0.31 \pm 0.48^{\mathrm{a}}$ & $0.69 \pm 0.95$ & $2.56 \pm 2.76$ & $0.81 \pm 0.83^{\mathrm{a}}$ & $5.01 \pm 5.32$ \\
\hline$<6 \mathrm{~mm}^{2}(\mathrm{n}=5)$ & $1.66 \pm 1.91$ & $4.6 \pm 9.7$ & $0.90 \pm 1.66$ & $0.70 \pm 1.25^{\mathrm{a}}$ & $0.20 \pm 0.42^{\mathrm{a}}$ & $0.40 \pm 0.84$ & $1.30 \pm 2.50$ & $0.50 \pm 0.85$ & $2.70 \pm 4.9$ \\
\hline$>6 \mathrm{~mm}^{2}(\mathrm{n}=6)$ & $6.64 \pm 2.87^{b}$ & $14.6 \pm 10.8^{c}$ & $2.50 \pm 1.38^{\mathrm{b}}$ & $2.83 \pm 0.41^{\mathrm{e}}$ & $0.67 \pm 0.52$ & $1.17 \pm 0.98^{\mathrm{b}, \mathrm{e}}$ & $4.67 \pm 1.75^{\mathrm{e}}$ & $1.67 \pm 0.52^{\mathrm{d}}$ & $8.84 \pm 3.43^{\mathrm{e}}$ \\
\hline
\end{tabular}

${ }^{\mathrm{a}} \mathrm{p}<0.05$.

$\mathrm{b}_{\mathrm{p}}<0.01$.

${ }^{c} \mathrm{p}<0.01$ between controls and IBN.

${ }^{\mathrm{d}} \mathrm{p}<0.05$.

${ }^{\mathrm{e}} \mathrm{p}<0.01$ within stratified subgroups in IBN-treated animals.

$\mathrm{A}=$ Estimated tumor area; $\mathrm{TC}=$ cortical infiltration; $\mathrm{TM}=$ infiltration of bone marrow; $\mathrm{TE}=$ infiltration of epiphysis; $\Sigma \mathrm{T}=$ sum of all topographical parameters; $\mathrm{Q}=$ qualitative tumor growth; $\Sigma$ score = total score sum $(\mathrm{A}+\mathrm{T}+\mathrm{Q}) ; \mathrm{PBS}=$ phosphate-buffered saline; IBN = ibandronate .

dard deviation (SD). Between-group comparisons were performed using the Student's t-test after testing for normal distribution of values, and $\mathrm{p}<$ 0.05 was considered significant. Correlations between parameters were calculated as simple Spearman correlation coefficients.

\section{Results}

In both treatment groups, 1 animal died prematurely and was excluded from analyses.

\section{Conventional Quantification}

OAs (X-CBA) were visible in all inoculated animals on day 18 p.i.. In the IBN treatment group, the OA increased, remained stable, or decreased over the 12-day treatment period, whereas in the controls, a constant increase of OA was observed until study termination. Following stratification for initial OA on day 18 p.i., a qualitative assessment revealed the possibility of a relationship between initial OA and treatment response. This was reflected in the corresponding MA on day 30 p.i. [8].

\section{Semiquantitative Analysis}

The total score sum $(\Sigma)$ was similar when both groups were analyzed without further stratification $(5.76 \pm 3.91$ vs. $5.01 \pm$ 5.52 for control and IBN-treated animals, respectively). However, as previously described [8], smaller tumor deposits were more likely to respond to treatment with IBN than larger lesions. We therefore applied an empirical cut-off of $6 \mathrm{~mm}^{2}$ to further stratify groups by initial OA as determined by X-CBA on day 18 p.i.. On average, IBN-treated animals with lesions $<6 \mathrm{~mm}^{2}$ had a significantly lower score sum than animals with lesions $>6 \mathrm{~mm}^{2}(2.70 \pm 4.99$ vs. $8.84 \pm 3.43$, respectively; $\mathrm{p}<0.01)$. When control animals were similarly stratified, no significant differences in score sum were observed $(\mathrm{p}=0.752)$. Mean score values for single parameters and stratification are shown in table 2. The extent of cortical infiltration (TC) showed no difference between the control and IBN-treated animals $(1.76 \pm 1.03$, controls vs. $1.50 \pm 1.46$, IBN-treated animals). Again, after stratification for initial OA, the IBN-treated animals showed a significantly lower TC when OAs were $<6 \mathrm{~mm}^{2}$ on day 18 p.i. $\left(0.70 \pm 1.25,<6 \mathrm{~mm}^{2}\right.$ vs. $2.83 \pm 0.41$, $\left.>6 \mathrm{~mm}^{2} ; \mathrm{p}<0.01\right)$. This difference in cortical infiltration was also seen between IBN-treated animals and the controls for osteolyses $<6 \mathrm{~mm}^{2}$ mainly reflecting the better drug response of smaller tumor deposits $\left(0.70 \pm 1.25, \mathrm{IBN}<6 \mathrm{~mm}^{2}\right.$ vs. $1.50 \pm$ 1.18 , controls $\left.<6 \mathrm{~mm}^{2} ; \mathrm{p}<0.05\right)$.

The evaluation of bone marrow infiltration (TM) showed a significant decrease after IBN treatment $(0.31 \pm 0.48$, IBN animals vs. $0.71 \pm 0.47$, controls; $p<0.05$ ). This effect was also observed after stratification of initial $\mathrm{OA}$ in the smaller lesions $\left(0.20 \pm 0.42, \mathrm{IBN}<6 \mathrm{~mm}^{2}\right.$ vs. $0.60 \pm 0.52$, controls $<6 \mathrm{~mm}^{2}$; $\mathrm{p}<0.05)$ and was absent when comparing greater $\left(>6 \mathrm{~mm}^{2}\right)$ metastases.

Epiphyseal infiltration (TE) did not differ between treated animals and controls $(0.69 \pm 0.95$, IBN vs. $0.47 \pm 0.80$, control animals). However, after stratification for initial OA, a significantly broader affection of the epiphysis in the IBN-treated animals was seen when tumor lesions were $>6 \mathrm{~mm}^{2}$ compared with the smaller lesions $\left(1.17 \pm 0.98\right.$, lesions $>6 \mathrm{~mm}^{2}$ IBN vs. $0.40 \pm 0.84$, lesions $\left.<6 \mathrm{~mm}^{2} \mathrm{IBN} ; \mathrm{p}<0.01\right)$. This increased epiphyseal infiltration in IBN-treated animals with greater tumor 
Table 3. Significant correlations ( $r$ values; all $p<0.001$ ) between semiquantitative score and quantitative histological $(\mathrm{H})$ and radiographic $(\mathrm{X})$ results

\begin{tabular}{llllll}
\hline \multirow{2}{*}{ Score } & \multicolumn{2}{l}{ Radiography $(\mathrm{X}-\mathrm{CBA})$} & & \multicolumn{2}{l}{ Histology (H-CBA) } \\
& Control & IBN & & Control & IBN \\
\hline $\mathrm{A}$ & 0.823 & 0.805 & & 0.702 & 0.970 \\
$\Sigma \mathrm{T}$ & 0.752 & 0.862 & & 0.753 & 0.927 \\
$\Sigma$ (total) & 0.845 & 0.854 & & 0.762 & 0.951 \\
Histology (H-CBA) & 0.705 & 0.970 & & - & - \\
\hline
\end{tabular}

IBN $=$ Ibandronate CBA $=$ computer-based analysis; $\Sigma \mathrm{T}=$ sum of all topographical parameters.

deposits remained significantly higher when compared with the greater lesions in untreated controls $(1.17 \pm 0.98$, lesions $>6 \mathrm{~mm}^{2}$ IBN vs. $0.43 \pm 0.79$, lesions $>6 \mathrm{~mm}^{2}$ in controls; $\mathrm{p}<0.01)$.

The sum of $\mathrm{T}$ indices $(\Sigma \mathrm{T})$ did not reflect the IBN treatment compared with untreated controls. It tended to show a higher score in the IBN-treated animals when stratification for initial OA was applied, but was not statistically significant (table 2). The quality of metastatic growth pattern in terms of infiltration or delineation $(\mathrm{Q})$ showed significantly better delineated tumor lesions in the IBN-treated animals compared with more invasive metastases in the untreated controls (means $0.81 \pm$ 0.83 , IBN vs. $1.38 \pm 0.72$, controls; $p<0.05$ ). This effect was absent after stratification for OA on day 18 . However, since lesions $<6 \mathrm{~mm}^{2}$ did respond significantly better to IBN treatment than bigger metastases (means $0.50 \pm 0.85$ vs. $1.67 \pm 0.52$, respectively), the effect of the unstratified data is mainly caused by the smaller lesions.

\section{Correlation between Score and Quantitatively Assessed Parameters (OA and MA)}

The total score sum $(\Sigma)$ significantly correlated with absolute MA for both PBS- $(\mathrm{r}=0.762 ; \mathrm{p}<0.001)$ and IBN-treated animals $(r=0.951 ; p<0.001)$. Combined analysis of both groups also revealed significant covariation (for MA, $\mathrm{r}=0.870$; $\mathrm{p}<0.001$; for OA $\mathrm{r}=0.862 ; \mathrm{p}<0.001$ ). This significant correlation was also found when OAs were compared with the total score sum in both groups $(\mathrm{r}=0.845$, controls and $\mathrm{r}=0.854$, IBN; $\mathrm{p}<0.001$ for both) (table 3).

Further stratification for the assessed score subparameters also showed a close correlation between tumor area (A) and the CBA-assessed OA (figs. $2 \mathrm{a}$ and b) and MA (figs. $2 \mathrm{c}$ and d). The quantitatively assessed MA estimated the score area and reflected the wide range of tumor areas in tibias from both groups. The close correlation between A and H-CBA ( $\mathrm{r}=$ 0.702 , controls and $r=0.970$, IBN-treated animals; $p<0.001$ ) remained after stratification for initial OA on day 18 p.i.

Bone marrow and epiphyseal infiltration for controls (means $0.65 \pm 0.49$ and $0.47 \pm 0.80$, respectively) and IBN-treated animals (means $0.38 \pm 0.50$ and $0.69 \pm 0.95$, respectively) showed

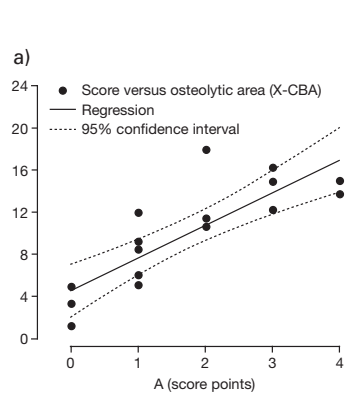

Osteolytic area
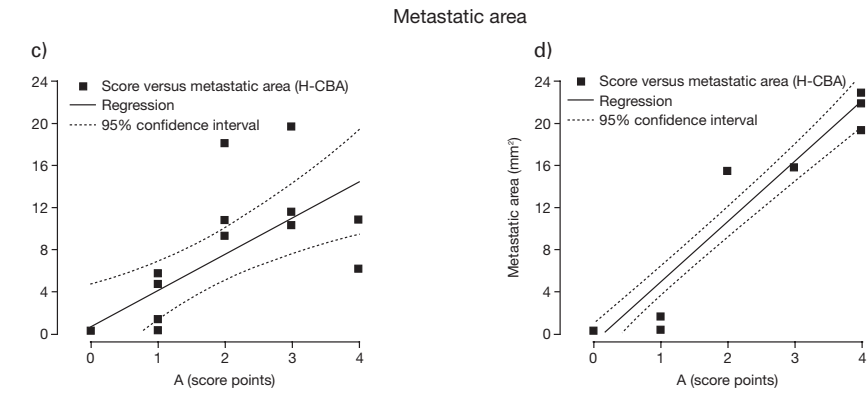

Fig. 2. Correlation of semiquantitative and quantitative histological tumor assessment. The estimated tumor area (A) and the quantitatively assessed metastatic (MA) and osteolytic areas (OA) determined by computer-based analyses correlate closely in both control and IBN-treated animals ( $\mathrm{n}=16$ bones/treatment group). Apparently missing data points are due to multiple projection of a single data point. Osteolytic area: (a) control animals, (b) IBN-treated animals; Metastatic area: (c) control animals, (d) IBN-treated animals. For correlations see table 3.

no correlation to OA or MA on day 30 p.i. (correlation coefficient (r) ranged from -0.238 to $-0.437 ; \mathrm{p}<0.01$ ). Presence and extent of epiphyseal infiltration appeared to reflect the local situation in more detail, but did not significantly contribute to the extent of measured OA and MA. As expected, the infiltration of bone marrow did not correlate with quantified tumor areas on radiographs (OA). Assessment of invasive or well delineated tumor growth as a qualitative aspect of the metastatic aggressiveness (Q) on histological sections was also not correlated with OA or MA. Comparison of the quantitatively assessed MA and OA were significantly correlated in both PBS- $(\mathrm{r}=0.705 ; \mathrm{p}<0.001)$ and IBN-treated animals $(\mathrm{r}=0.970$; $\mathrm{p}<0.001)$.

To focus on the additional information on metastatic tumor growth that is provided by the score, the data were normalized in order to eliminate the influence of MA. As a consequence, the close correlation between histological assessment and the score was reduced to $r=0.362$. Therefore, it is most likely that the score provides additional information on top of MA, but to which extend cannot be proofed upon the presented data.

\section{Discussion}

The aim of the present study was to develop a semiquantitative tool to assess and stage bone lesions in a nude rat model 
of MBD. The key criteria were to fill the gap between comprehensive (but labor-intensive) approaches and simple scoring systems [5-7]. Overall, this score analysis showed good correlation with established techniques, suggesting that this approach can be adopted in the experimental setting to measure the extent of bone metastases. Although some individual indices did not correlate when considered in isolation, these still warrant inclusion as they not only contribute to the overall score sum but are likely to provide additional descriptive detail in the experimental setting.

In general, a drawback to histological assessments is a relatively low sensitivity for osteolytic lesions. Here, we report a lower detection limit of approximately $1.3 \mathrm{~mm}^{2}$. In the experimental setting, sensitivity could be increased by preparing a greater number of sections per bone. Although a greater number of sections will reduce the lower detection limit, this needs to be balanced against the price of higher resource utilization. Furthermore, the rate of apparent false-negative histological sections in this study is likely due to the small number of sections per bone. The value and significance of histological analyses is not the detection of small tumor lesions but the confirmation or exclusion of other lesion characteristics. As histological approaches are not usually used as a method for screening bone lesions, comparison with radiological assessments at this stage would yield limited benefits. In this study, 3 sections per bone provided sufficient tissue to perform detailed evaluation of metastatic involvement. This suggests that this new score is likely to reduce the need for time-consuming multiple bone sections.

Radiographic analyses of experimental tumor osteolyses have been frequently employed [6]. There is uncertainty, however, whether this technique allows full evaluation of tumor mass and extent in bone. For example, using radiographic tumor analysis, animals treated with antiresortive therapy (i.e., a bisphosphonate like IBN) can erroneously result in apparently stable bone lesions. Using radiography, it is not possible to differentiate between osteoclast inhibition or a reduction or inhibition of the tumor growth itself.

The data presented in this study suggest that the total score sum $(\Sigma)$ can predict OA on radiographs and that this may provide an alternative measure. Our data also suggests that stratification of data, based on baseline tumor size may be essential when using the score. Conventional radiographs provide a summation of all histological-anatomically affected structures. In this study, radiographic analysis had a higher sensitivity for detection of bone metastases compared with the histological analysis. When small bone lesions are present, this approach may have practical benefits.

This new score proved useful in providing more detailed information on the pattern of metastatic growth and the action of a bisphosphonate by detecting differences in lesion status between IBN- and PBS-treated animals. In contrast to control animals $(100 \%$ of animals had an increase in OA), $81 \%$ of IBN-treated animals had either stable or decreased metastatic burden (1 IBN-treated animal achieved complete remission, both on radiograph and histological sections). IBN treatment significantly reduced the extent of bone marrow infiltration (TM) and cortical infiltration (TC) in lesions $<6 \mathrm{~mm}^{2}$. Furthermore, of particular note is the significant decrease in metastatic aggressiveness (Q). This suggests the infiltration pattern after IBN treatment is better delineated and not as invasive as in the untreated controls. In contrast, epiphyseal infiltration was greater in the IBN treatment group when initial OA size was $>6 \mathrm{~mm}^{2}$. We speculate that this might be an effect of the growing skeleton in adolescent rats; although tumor cell adhesion is likely to be reduced on bisphosphonate-treated bone matrix, this is absent in the cartilage of the growing epiphysis. This finding cannot, therefore, be directly translated into the clinical situation in mature human patients with calcified bone, but is worth investigating further in aged rats.

The dose of IBN was obtained from another study in rats, in which IBN significantly inhibited tumor-related bone destruction [10]. Comparable results were expected in the present study thus allowing the assessment of the new score system in an effective therapeutic setting. Although animal studies can provide insight into efficacy or mode of action, a direct translation of the applied dose into the clinical situation requires clinical trials.

This study has certain limitations, for example, we only analyzed a limited number of time points. However, this is unlikely to affect the validity of the score as it is based on the assessment of histological and anatomical structures which are present throughout all stages of metastatic growth. We also recognize that osteoblastic lesions were not assessed during this study. Although yet to be confirmed, we would expect this score to be of use when examining osteoblastic lesions (and indeed mixed lesions) as these too have measurable histological characteristics which are discernable from surrounding healthy bone. Although the correlation with radiographic scores will need further clarification (due to differences in radiolucency with osteoblastic growth) it will still be possible to evaluate tumor area, infiltration, and growth patterns in osteoblastic models. As only one bone lesion model, this raises the question of whether it would apply to other animal models or tumor cell lines. Although this clearly will need confirmation by additional studies, we fully expect the score to be transferable to other laboratory animal models as anatomical and histological features of the skeleton are similar. Similarly, we predict that the score will apply to other tumor cell lines as it is the extent and pattern of bone destruction which is being measured as a result of inoculation, not a direct characteristic of the cancer cells themselves.

It should be noted that this scoring system does not measure total tumor burden. This may limit its use to those cancer models in which focal lesions have been either previously identified or, as in this study, generated by focal inoculation. This score was designed to be used primarily in conjunction with focal inoculation targeting the skeleton only. Measuring 
overall tumor burden (e.g., visceral metastases) was not an objective when designing this score.

In conclusion, our novel semiquantitative histological score provides a rapid and reliable means to assess the extent of metastatic bone involvement in the laboratory. This score can provide more detailed information on metastatic tumor cell infiltration of bone that is not captured by conventional measures of tumor burden. It also offers an alternative when radiographic analytical methods are unavailable or inapplicable (i.e., when tumor destruction of bone is significant). While the score does not provide the high-resolution detail of 3-dimensional volumetric measurement, it has value as an accurate, rapid, and conveniently applicable assessment tool. The scoring system was also sensitive to histological changes in bone following treatment with a bisphosphonate. Significant reduction of bone marrow and cortical infiltration following exposure to IBN suggests that local control of metastases can be detected, as shown by reduced tumor cell infiltration and greater definition and delineation of the overall lesion area.

\section{Acknowledgements}

The authors wish to thank Stefan Hoert, Martina Wagner, Matthias Metz, and Johanna Meixner for their excellent technical assistance during the animal experiments and histological preparations. This work was supported by a grant from the Medical Faculty, University of Heidelberg (MS 76/99). The authors certify that they have not entered into any agreement that could interfere with their access to the data on the research, nor upon their ability to analyze the data independently, to prepare manuscripts, and to publish them. Professional medical writing assistance was received during the preparation of this manuscript.

\section{Abbreviations}

$\mathrm{CBA}=$ Computer-based analysis; $\mathrm{H}-\mathrm{CBA}=$ histologic $\mathrm{CBA} ; \mathrm{X}-\mathrm{CBA}=$ radiographic CBA; EDTA = ethylenediaminetetraacetic acid; IBN = ibandronate; $\mathrm{MA}=$ metastatic area; $\mathrm{MBD}=$ metastatic bone disease $; \mathrm{OA}=$ os teolytic area; p.i. $=$ post-inoculation; $\mathrm{PBS}=$ phosphate-buffered saline . Sum $=\Sigma ;$ Tumor Area $=\mathrm{A} ; \mathrm{TC}=$ topographic localization to cortical bone; $\mathrm{TM}=$ topographic localization to bone marrow; $\mathrm{TE}=$ topographic localization to epiphyseal growth plate; $\mathrm{Q}=$ quality of infiltration pattern; $\mathrm{SD}=$ standard deviation

\section{References}

1 Coleman RE, Rubens RD: Bone metastases and breast cancer. Cancer Treat Rev 1985;12:251-270.

2 Coleman RE, Rubens RD: The clinical course of bone metastases from breast cancer. Br J Cancer 1987;55:61-66.

3 Theriault RL, Hortobagyi GN: Bone metastasis in breast cancer. Anticancer Drugs 1992;3:455-462.

4 Russell RG: Bisphosphonates: from bench to bedside. Ann NY Acad Sci 2006;1068:367-401.

5 Krempien B, Wingen F, Eichmann T, Mueller M, Schmahl D: Protective effects of a prophylactic treatment with the bisphosphonate 3-amino-1-hy droxypropane-1.1-bisphosphonic acid on the development of tumor osteopathies in the rat: experimental studies with the Walker carcinosarcoma 256. Oncology 1988;45:41-46.
6 Sasaki A, Boyce BF, Story B, Wright KR, Chapman M, Boyce R, Mundy GR, Yoneda T: Bisphosphonate risedronate reduces metastatic human breast cancer burden in bone in nude mice. Cancer Res 1995;55:3551-3557.

7 Kurth AA, Muller R: The effect of an osteolytic tumor on the three-dimensional trabecular bone morphology in an animal model. Skeletal Radiol 2001;30:94-98.

8 Neudert M, Fischer C, Krempien B, Bauss F, Seibel MJ: Site-specific human breast cancer (MDA-MB231) metastases in nude rats: model characterisation and in vivo effects of ibandronate on tumour growth. Int J Cancer 2003;107:468-477.
9 Cailleau R, Young R, Olive M, Reeves WJ Jr: Breast tumor cell lines from pleural effusions. J Natl Cancer Inst 1974;53:661-674.

10 Juraschek M, Seibel MJ, Woitge HW, Krempien B, Bauss F: Association between histomorphometry and biochemical markers of bone turnover in a longitudinal rat model of PTHrP-mediated tumor osteolysis. Bone 2000;26:475-483.

11 Orr W: Tumor-bone interaction in skeletal metastases. Clin Orthop 1995;312:19-33. 